\title{
Musculoskeletal (MSK) and Sport and Exercise Medicine (SEM) in General Practice (GP): A Novel GP-based MSK and SEM Clinic for Managing Musculoskeletal symptoms in a GP
}

Neil Heron

NHS, Northern Ireland

\begin{abstract}
Musculoskeletal (MSK) complaints are common within primary care (1) (2) (3) but some General Practitioners (GPs)/family physicians do not feel comfortable managing these symptoms (3), preferring to refer onto hospital specialists or Integrated Clinical Assessment and Treatment Services (ICATs). Long waiting times for hospital outpatient reviews are a major cause of patient inconvenience and complaints (4). We therefore aimed to establish a GP-ran MSK and sport and exercise medicine (SEM) clinic based within a Belfast GP surgery that would contribute to a sustainable improvement in managing these common conditions within primary care as well as reducing waiting times for patients with these conditions to see a specialist. This shift from hospital-based to community-based management is in-keeping with recent policy changes within the UK health-system, including Transforming Your Care within Northern Ireland (NI) (5). The GP-ran MSK and SEM clinic was held monthly within a Belfast GP practice, staffed by one GP with a specialist interest in MSK and SEM conditions and its performance was reviewed over a three month period. Parameters audited included cases seen, orthopaedic and $\mathrm{x}$-ray referral rates and secondary care referrals comparing the GP practice's performance to the same time period in the previous year as well as patient satisfaction questionnaires.
\end{abstract}

\section{Problem}

MSK complaints are common within primary care (1) (2) (3) but some GPs do not feel comfortable managing these symptoms (3), preferring to refer onto hospital specialists or Integrated Clinical Assessment and Treatment Services (ICATs). Patient dissatisfaction with waiting times is a major cause of complaints within healthcare systems (4). The current delivery of the UK health system is in a state of flux with the emphasis now on communitybased management of health issues rather than hospital-focused approaches. Indeed within Northern Ireland, for example, the Transforming Your Care policy is promoting more community-based management options for patients rather than the traditional hospitalbased (5). As a direct result, commissioning a MSK and SEM clinic within a local GP clinic was deemed as a high priority area for improvement and was motivated by a desire to provide:

"..... the right care in the right place at the right time."

The aim of this quality improvement project was therefore to develop a reproducible GP-staffed MSK and SEM clinic based within primary care that is economically sound and sustainable, providing high-patient satisfaction.

\section{Background}

With approximately twenty per cent of GPs'/family physicians' workload being related to MSK conditions (1) (2) (3), GPs and primary care staff need to have access to appropriate clinics for investigating and managing MSK conditions. Moreover primary care staff commonly report low confidence levels in managing common
MSK conditions (3), further increasing the need for specialist MSK and, a related discipline, SEM knowledge within GP. The current health system within the UK dictates that patients with MSK/SEM complaints are initially reviewed by the GP and then referred onto secondary care when further investigation and management is required. The secondary care options would include an appointment in hospital with an orthopaedic specialist or review within orthopaedic ICATs typically within a community-based healthcare centre. However waiting times for these specialities are long and waiting times for elective care is a common source of patient dissatisfaction within healthcare systems (4). Alternatively, if GPs had appropriate specialist knowledge in this area then the patients' management could largely be within the local GP practice with secondary care input only for a select few patients not able to be managed appropriately by the GP, for example when surgery is required, consequently decreasing the waiting times for elective care in hospitals. This latter approach would be supported by the Transforming Your Care policy in NI, with a shift in emphasis now from hospital-based to community-based management options, allowing the delivery of healthcare as close to the patients' home as practical (5). The ICAT service model has illustrated that GPs with a specialist interest can manage common musculoskeletal complaints competently but this project is developing this idea further by demonstrating that GPs with a specialist interest can manage these patients within their own practices.

See supplementary file: ds4719.doc - "Outline 5 with references"

\section{Baseline measurement}

The referral rates for the practice was reviewed for the period 
BMJ Quality Improvement Reports

August to October 2013. There was 881 referrals overall from the GP practice to secondary care, with fifty-five specific orthopaedic referrals. Over this period there was 317 musculoskeletal presentations within the practice. The number of physiotherapy referrals between August to October 2013 was 133 and the number of radiology referrals for $x$-rays was 319 . Thirteen patients were referred to rheumatology from the practice during this period. A review of the 2013 orthopaedic ICAT waiting times showed that 5,833 patients $(49.5 \%)$ were seen between $0-6$ weeks of referral, 2,304 patients (19.6\%) between 6 - 9 weeks, 1,872 patients (15.9\%) waiting between $9-12$ weeks, 1,389 patients (11.8\%) waiting between $12-15$ weeks, 236 patients (2\%) $15-18$ weeks and 144 patients (1.2\%) waiting more than 18 weeks to be seen (from a total of 11,778 patients). The cost of a routine hospital orthopaedic outpatient review in 2014 was $£ 213$ and the average orthopaedic ICAT cost per attendance was $£ 82$.

\section{Design}

This quality improvement project was conducted in a Belfast GP practice of approximately 9,000 patients and five GP partners. The GP practice uses EMIS electronic patient records and is based approximately two miles from Belfast city centre. The practice introduced a MSK and SEM clinic staffed by one GP with a specialist interest in MSK and SEM conditions, with appropriate post-graduate qualifications in these areas, and its performance was reviewed over a three month period between August 2014 to October 2014. One monthly four hour clinic was held over this period and appointment times were approximately twenty minutes. All primary care staff within the practice could refer to the clinic any patient with MSK and/or SEM presentations, on whom they wanted some specialist input. Management options included injection therapy, exercise prescription and onward referrals to appropriate colleagues, for example physiotherapy.

The practice's performance during the running of the clinic was compared to the same time period in 2013 to control for other variables outwith the introduction of the GP-based MSK and SEM clinic. The variables which we were interested in monitoring included cases seen in the clinic, waiting times, treatments given, and onward referral to colleagues from the clinic.

Equipment which was available to the GP within the GP-based MSK and SEM clinic included a clinical room with a computer to allow access to the patients' GP clinical records, a couch and lighting. There were also facilities to undertake joint injections under appropriate sterile conditions. Within the primary care clinic, there was access to laboratory blood tests (to exclude an inflammatory condition) and imaging, including open access radiology for plain films. If needed, patients could be referred for input to orthopaedic ICATs and hospital specialities, including rheumatology and orthopaedics, using the typical referral methods. A random sample of ten patients were selected to complete a patient satisfaction questionnaire (Appendix 1). This questionnaire was developed from a recognised patient satisfaction questionnaire for assessing GP services (6), with input from the Patient Experience Group at the Royal Group of Hospitals, Belfast.

\section{Strategy}

Thirty-five patients were seen in the GP-staffed MSK and SEM clinic, fourteen males and twenty-one females, between August and October 2014. The age range of patients seen was from thirty-five to seventy seven years old. The patients were generally referred from the other GPs within the practice but a minority of referrals were also sent from physiotherapy, podiatry and hospital colleagues. For example, one patient was referred to the clinic from rheumatology due to the long waiting times for joint injections within their department. The thirty-five patients seen are included in Table 1.

With regards management in the GP-based clinic, as well as the steroid injection, the patients were also given advice regarding appropriate conservative management options, including analgesia, muscle stretches and strengthening exercises. The commonest management option employed within the clinic was steroid injection. A source of appropriate patient information used within the clinic was from Arthritis Research UK (ARUK), who provide information leaflets for patients on various musculoskeletal complaints (7). The main joint presenting to the clinic was shoulder, with the main pathology detected here being within the supraspinatus muscle (Table 2).

Ten participants were randomly selected from the three clinics held to complete a patient satisfaction questionnaire (Appendix 1). This included four males and six females of mean age fifty-one years old (to the nearest year). Their responses are summarised in Table 3. The patients generally felt that it was easy to get an appointment at the GP-based clinic, with the investigating doctor generally doing enough tests and patients had high levels of confidence in the treating GP. Communication at the clinic was clear, with high patient satisfaction with the services offered. Patients appeared happier to have their conditions managed within their GP surgery than compared to hospital or another community-based health facility and would prefer to see future specialists within their GP clinic rather than outwith. There was also a space at the bottom of the questionnaire for free text comments and three different people left a comment, included in Appendix 2. The patients comments illustrated how highly they valued the service at the GP clinic.

The practice referral rates between August to October 2013 and 2014 are included in Table 4. The number of onward referrals made by the treating GP following been seen within the MSK and SEM clinic in the period August to October 2014 was three - two to physiotherapy and one to rheumatology, to exclude an inflammatory cause of the patient's pain. This is compared to the thirty-five referrals which would have been made from the practice if this MSK and SEM clinic did not exist. Comparing referral rates between August to October 2013 and the same time period in 2014, overall referrals from the practice were reduced by 147 , orthopaedic referrals were reduced by 2 , whilst rheumatology referrals were reduced by 3, MSK presentations to the practice were reduced by 60 , and physiotherapy and $\mathrm{x}$-ray referrals were reduced by 47 and 90 , respectively.

All the patients referred to the GP-based MSK clinic were seen 
within four weeks. A review of the 2013 orthopaedic ICAT waiting times showed that 5,833 patients $(49.5 \%)$ were seen between $0-6$ weeks of referral, 2,304 patients (19.6\%) between 6 - 9 weeks, 1,872 patients (15.9\%) waiting between $9-12$ weeks, 1,389 patients (11.8\%) waiting between $12-15$ weeks, 236 patients (2\%) $15-18$ weeks and 144 patients (1.2\%) waiting more than 18 weeks to be seen (from a total of 11,778 patients). The cost of a routine hospital orthopaedic outpatient review in 2014 was $£ 213$ and the average orthopaedic ICAT cost per attendance was £82 (information obtained through direct communications with the finance department of DHSSPS (NI)). The cost of one hour of GPpatient contact, including direct care staff costs with qualification costs, for 2013-14 was £183 (information obtained through direct communications with the finance department of DHSSPS (NI)). Three patients were at least seen per hour and therefore the cost per patient reviewed at the GP-based MSK and SEM clinic was conservatively costed at $£ 61$ per patient. Therefore if all the clinic's patients were reviewed within a hospital orthopaedic outpatient clinic the cost to the Northern Ireland health service would have been $£ 7,455$ or within the orthopaedic ICAT system the cost would have been $£ 2,870$. This is compared to the $£ 2,135$ which it cost to run the GP-based MSK and SEM clinic, a potential saving of between $£ 735$ and $£ 5,320$ per thirty-five patients reviewed.

See supplementary file: ds4720.doc - "Outline 5 with references"

\section{Post-measurement}

Thirty-five patients were seen in the GP-staffed MSK and SEM clinic, fourteen males and twenty-one females, between August and October 2014. The age range of patients seen was from thirty-five to seventy seven years old. The patients were generally referred from the other GPs within the practice but a minority of referrals were also sent from physiotherapy, podiatry and hospital colleagues. For example, one patient was referred to the clinic from rheumatology due to the long waiting times for joint injections within their department. The thirty-five patients seen are included in Table 1.

With regards management in the GP-based clinic, as well as the steroid injection, the patients were also given advice regarding appropriate conservative management options, including analgesia, muscle stretches and strengthening exercises. The commonest management option employed within the clinic was steroid injection. A source of appropriate patient information used within the clinic was from Arthritis Research UK (ARUK), who provide hand-outs for patients on various musculoskeletal complaints (7). The main joint presenting to the clinic was shoulder, with the main pathology detected here being within the supraspinatus muscle (Table 2).

Ten participants were randomly selected from the three clinics held to complete a patient satisfaction questionnaire (Appendix 1). This included four males and six females of mean age fifty-one years old (to the nearest year). Their responses are summarised in Table 3.

The patients generally felt that it was easy to get an appointment at the GP-based clinic, with the investigating doctor generally doing enough tests and patients had high levels of confidence in the treating GP. Communication at the clinic was clear, with high patient satisfaction with the services offered. Patients appeared happier to have their conditions managed within their GP surgery than compared to hospital or another community-based health facility and would prefer to see future specialists within their GP clinic rather than outwith.

There was also a space at the bottom of the questionnaire for free text comments and three different people left a comment. A fortynine year old male wrote:

"(The service is) a great asset to the surgery."

Another forty-nine year old male wrote:

"(The doctor) made me feel at ease and talked to me like a friend. I would not hesitate to attend his clinic again."

A fifty-five year old female also commented:

"Excellent service."

The number of onward referrals made by the treating GP following been seen within the MSK and SEM clinic in the period August to October 2014 was three - two to physiotherapy and one to rheumatology, to exclude an inflammatory cause of the patient's pain. This is compared to the thirty-five referrals which would have been made from the practice if this MSK and SEM clinic did not exist.

Between August 2014 to October 2014 there was 732 referrals overall from the GP practice, with fifty-three specific orthopaedic referrals. Over this period there was 257 MSK presentations within the practice. The number of physiotherapy referrals between August to October 2014 was eighty-six from the practice and the number of radiology referrals for $\mathrm{x}$-rays between August to October 2014 was 229 . Ten patients were referred to rheumatology from the practice over this period. All the patients referred to the GP-based MSK clinic were seen within four weeks. The cost of one hour of GPpatient contact, including direct care staff costs with qualification costs, for 2013-14 was £183. Three patients were at least seen per hour and therefore the cost per patient reviewed at the GP-based MSK and SEM clinic was conservatively costed at £61 per patient.

These referral rates were compared to the same time period for the practice in 2013 when there was 881 referrals overall from the GP practice, with fifty-five specific orthopaedic referrals. Over this period there was 317 musculoskeletal presentations within the practice. The number of physiotherapy referrals between August to October 2013 was 133 and the number of radiology referrals for $x$ rays was 319 . Thirteen patients were referred to rheumatology from the practice during this period. A review of the 2013 orthopaedic ICAT waiting times showed that 5,833 patients $(49.5 \%)$ were seen between 0-6 weeks of referral, 2,304 patients (19.6\%) between 6 9 weeks, 1,872 patients (15.9\%) waiting between $9-12$ weeks, 1,389 patients (11.8\%) waiting between $12-15$ weeks, 236 patients $(2 \%) 15-18$ weeks and 144 patients (1.2\%) waiting more than 18 weeks to be seen (from a total of 11,778 patients). The cost of a routine hospital orthopaedic outpatient review in 2014 was 
$£ 213$ and the average orthopaedic ICAT cost per attendance was £82. Therefore if all the clinic's patients were reviewed within a hospital orthopaedic outpatient clinic the cost to the Northern Ireland health service would have been $£ 7,455$ or within the orthopaedic ICAT system the cost would have been $£ 2,870$. This is compared to the $£ 2,135$ which it cost to run the GP-based MSK and SEM clinic, a potential saving of between $£ 735$ and $£ 5,320$ per thirty-five patients reviewed. These referral rates are included in Table 4.

\section{Lessons and limitations}

The review of referral rates from the GP practice is dependent on the primary care staff coding appropriately and our statistics are therefore limited by the quality of the information which the staff enter into the system. This may therefore lead to under- or overestimating referral rates.

This study was performed in one GP practice which allowed us $100 \%$ follow-up of our patients. The next stage for the quality improvement project will be to replicate these findings within a larger geographical area and then present the findings to local commissioners to further extend this trial across different areas of the UK.

\section{Conclusion}

With the financial constraints now faced by the NHS and the new policies shifting focus from hospital-based to community-based management options, we present a novel service model for managing MSK and SEM complaints in primary care. This model can make sound economical sense and deliver high patient satisfaction within primary care, with low waiting times. We present a reproducible model that can be commissioned as a service by the local clinical commissioning groups and be extended throughout the $\mathrm{NI}$ health service as part of Transforming Your Care policy.

\section{References}

1. Jordan K, Clarke A, Symmons D, Fleming D, Porcheret M, Kadam U, et al. Measuring disease prevalence: a comparison of musculoskeletal disease using four general practice consultation databases. British Journal of General Practice 2007;57(534):7-14.

2. Jordan K, Kadam U, Hayward R, Porcheret M, Young C, Croft $P$. Annual consultation prevalence of regional musculoskeletal problems in primary care: an observational study. BMC musculoskeletal disorders 2010;11(144).

3. Goff I, Wise E, Coady D, Walker D. Musculoskeletal training: are GP trainees exposed to the right case mix for independent practice? Clinical Rheumatology 2014;Sept 6 (Epub).

4. Ringard Å, Sagan A, Sperre Saunes I, Lindahl A. Norway: health system review. Health Systems in Transition 2013;15(8):1-162.

5. Department of Health, Social Services and Public Safety Northern Ireland. Transforming Your Care - A Review of
Health and Social Care in Northern Ireland. Transforming Your Care Report 2011; Dec.

6. Grogan S, Conner M, Norman P, Willits D, Porter I. Validation of a questionnaire measuring patient satisfaction with general practitioner services. Quality in Health Care 2000;9(4):210-5.

7. Arthritis Research UK. Patient Information Leaflets. Arthritis Research UK website 2014.

8. Mahesh M, Durand DJ. The Choosing Wisely Campaign and Its Potential Impact on Diagnostic Radiation Burden. Journal of the American College of Radiology 2013 1;10(1):65-66.

9. Belfast Health and Social Care Trust. Annual Report and Accounts 2013/14 - Belfast Trust. Annual Report and Accounts 2013/14 2014;1(1):1.

10. Ahmed I, Gertner E. Safety of Arthrocentesis and Joint Injection in Patients Receiving Anticoagulation at Therapeutic Levels. Am J Med 2012 3;125(3):265-269.

11. Conway R, O'Shea F, Cunnane G, Doran M. Safety of joint and soft tissue injections in patients on warfarin anticoagulation.. Clinical Rheumatology 2013;32(12):1811-4.

12. Gillman LM, Kirkpatrick AW. Portable bedside ultrasound: the visual stethoscope of the 21st century. Scandinavian Journal of Trauma Resuscitation \& Emergency Medicine 2012 MAR 9;20:18.

13. A Hamid MS, Mohamed Ali MR, Yusof A, George J. Plateletrich plasma (PRP): an adjuvant to hasten hamstring muscle recovery. A randomized controlled trial protocol (ISCRTN66528592).. BMC musculoskeletal disorders 2012;13(138).

14. Ozturan KE, Yucel I, Cakici H, et al. Autologous blood and corticosteroid injection and extracoporeal shock wave therapy in the treatment of lateral epicondylitis. Orthopedics 2010;33(2):84-91.

15. Patel S, Dhillon MS, Aggarwal S, Marwaha N, Jain A. Treatment With Platelet-Rich Plasma Is More Effective Than Placebo for Knee Osteoarthritis: A Prospective, DoubleBlind, Randomized Trial. The American Journal of Sports Medicine 2013 February 01;41(2):356-364.

16. Filardo G, Kon E, Di Martino A, Di Matteo B, Merli M, Cenacchi $A$, et al. Platelet-rich plasma vs hyaluronic acid to treat knee degenerative pathology: study design and preliminary results of a randomized controlled trial.. BMC Musculoskeletal Disorders 2012;13(229).

17. Sánchez M, Guadilla J, Fiz N, Andia I. Ultrasound-guided platelet-rich plasma injections for the treatment of osteoarthritis of the hip.. Rheumatology 2012;51(1):144-50.

\section{Declaration of interests}

The main author is a practising GP with a specialist interest in musculoskeletal and sport and exercise medicine conditions.

\section{Acknowledgements}

I would like to acknowledge the STEP medical leadership 
BMJ Quality Improvement Reports

programme within the Belfast Trust, Northern Ireland, particularly Dr Claire Lundy and her team, for their help throughout this project. I would also like to acknowledge the help of Dr Nigel Hart and Prof Margaret Cupples with regards supervision. 